\title{
Synthesis and Real-Time Magnetic Manipulation of a Biaxial Superparamagnetic Colloid
}

\author{
Jin Young Kim, ${ }^{\dagger}$ Frank E. Osterloh, ${ }^{*}, \dagger$ Hiroki Hiramatsu, ${ }^{\dagger}$ R. K. Dumas, ${ }^{\ddagger}$ and Kai Liu ${ }^{*, *}$ \\ Departments of Chemistry and Physics, University of California-Davis, Davis, California 95616
}

Received: January 19, 2005; In Final Form: April 5, 2005

\begin{abstract}
Superparamagnetic colloidal plates were synthesized from tetrabutylammonium stabilized $\mathrm{Ca}_{2} \mathrm{Nb}_{3} \mathrm{O}_{10}$ nanosheets and oleic acid-stabilized $\mathrm{Fe}_{3} \mathrm{O}_{4}$ nanoparticles. Modification with 3-aminopropyltrimethoxysilane produces amine-terminated $\mathrm{Ca}_{2} \mathrm{Nb}_{3} \mathrm{O}_{10}$ with an amine concentration of $0.43 \pm 0.06$ groups per $\mathrm{Ca}_{2} \mathrm{Nb}_{3} \mathrm{O}_{10}$ unit as follows from spectroscopic quantification with trinitrobenzenesulfonic acid as a dye. Treatment of the modified sheets in THF/ethanol with $5.3 \mathrm{~nm}$ oleic acid-stabilized magnetite nanoparticles yields pseudo-2D assemblies that consist of $2 \mathrm{~nm}$ thick nanosheets decorated on both sides with a dense collection $\left(9.3 \pm 0.5 \times 10^{3}\right.$ particles per square micrometer per side) of magnetite particles. In noncoordinating or weakly coordinating solvents, these composite particles further aggregate into stacked aggregates with a mean edge length of $1.6 \pm 0.7 \mu \mathrm{m}$ and a thickness of $79 \pm 30 \mathrm{~nm}$. The colloidal plates were characterized by elemental analysis, X-ray powder diffraction, and infrared and UV/vis spectroscopy. SQUID measurements show that films of the aligned particles are superparamagnetic at room temperature. The magnetic hysteresis that is observed at $5 \mathrm{~K}$ reveals that the plates have a magnetic anisotropy with the easy axis in the plane of the plates and the hard axis perpendicular to it. Calculations show that the magnetic anisotropy is a direct consequence of the two-dimensional distribution of the magnetic nanoparticles on the sheets. Optical microscopy reveals that when suspended in ethanol or THF, the colloidal plates can be rotated in real time with a variable external magnetic field (200 Oe). Magnetic alignment of the particles in suspensions also produces asymmetric light scattering patterns and magnetic birefringence. These effects and the observed magneto-orientational properties make the biaxial colloids interesting as components in displays and as magnetic actuators.
\end{abstract}

\section{Introduction}

Nanoscience has made much progress in recent years in the synthesis of nanostructured materials and in the understanding of their physical properties. One of the current challenges in this area is to develop methods to control the motion and orientation of nano- and microscale structures in real-time. Guided motion plays a central role not only in the processes of life but also in many emerging technologies in engineering, medicine, biology, chemistry, and physics. Motors, pumps, valves, and certain kinds of sensors all rely on movable parts that undergo guided motions to accomplish their complex functions. Nano- and micrometer scale analogues of such devices are highly desirable because they promise improved performance and lower energy consumption as a result of their smaller sizes. ${ }^{1,2}$ However, the realization of such micromechanical devices is restricted by the techniques that are available today to externally control and drive nano- and microscale structures in real time. ${ }^{3-5}$ An efficient way to control the orientation of nano- and micromaterials is by application of a magnetic field. This requires the material to have a magnetic anisotropy $\Delta \chi=\chi_{\|}-\chi_{\perp}$, in which $\chi_{\|}$and $\chi_{\perp}$ are the susceptibilities along two perpendicular directions of the material. This requirement can be met for example by magnetic liquid crystals, a large variety of which have been synthesized over the past years. ${ }^{6}$ These metallomesogens often consist of molecules that contain lanthanide ions as paramagnetic components. In solution such molecules form orientationally ordered

\footnotetext{
Department of Chemistry.

$\doteqdot$ Department of Physics.
}

(nematic) or positionally ordered (smectic) phases that are characterized by a magnetic anisotropy, which makes it possible to align the crystals with a magnetic field on the order of 4-20 kOe. ${ }^{7-9}$ Due to their large number of paramagnetic centers, single domain inorganic nanoparticles can have effective magnetic moments 3-5 orders of magnitude larger than those of single metallic ions. As a consequence, such nanostructures react much more sensitively to magnetic fields and are much more amenable to magnetic manipulation. ${ }^{10,11}$ Lee et al. demonstrated recently that a 60 Oe field is sufficient to trap and orient magnetotactic bacteria (1.5-3.0 $\mu \mathrm{m}$ in length), which contain ferrimagnetic structures made of $\sim 50 \mathrm{~nm}$ magnetite nanoparticles in their cell interiors. ${ }^{12-14}$ Bentley et al. showed that ferromagnetic rods containing Ni ends $\left(13.0 \times 0.2 \mu \mathrm{m}^{2}\right)$ could be rotated in solution by application of a rotating magnetic field of $65-350$ Oe. ${ }^{15}$ These and similar magnetic nanostructures ${ }^{16}$ can be used potentially to transfer energy or momentum to a closed system, ${ }^{17}$ to manipulate the orientations and positions of diamagnetic nano- and micromaterials, and to drive microscale motors and valves.

Because of synthetic limitations, research in the field of magnetically addressable nanostructures and metallic mesogens has concentrated on pseudo-one-dimensional systems such as colloidal rods, nanowires, and calamitic mesogens (rodlike molecules). ${ }^{18,19}$ Due to their uniaxial geometry, these structures have one easy magnetic axis (see Figure 1A), which in the presence of an external magnetic field aligns with the field lines. ${ }^{20,21}$ In contrast, magnetic structures with platelike morphology (Figure 1B) can have multiple in-plane easy axes, which 

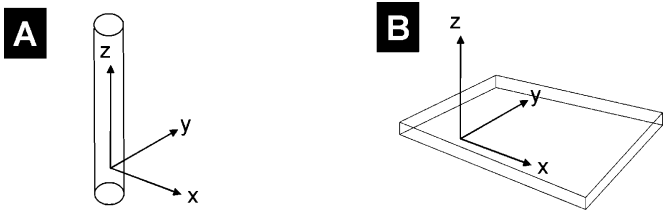

$\left|\chi_{z}\right|>\left|\chi_{x}\right| \approx\left|\chi_{y}\right|$

$\left|\chi_{x}\right| \approx\left|\chi_{y}\right| \gg\left|\chi_{z}\right|$

Figure 1. Magnetic susceptibilities of uniaxial (A) and biaxial (B) colloidal particles.

might enable magnetic manipulation of such structures in three dimensions with use of suitably oriented fields.

We recently discovered a facile synthetic pathway to colloidal pseudo-two-dimensional superparamagnetic microstructures from $\mathrm{Fe}_{3} \mathrm{O}_{4}$ and $\mathrm{Ca}_{2} \mathrm{Nb}_{3} \mathrm{O}_{10}$ nanoparticle building blocks. ${ }^{22,23}$ When suspended in organic solvents, these magnetic plates align with a magnetic field to produce strongly birefringent dispersions that are optically similar to magnetic liquid crystals. To understand the magnetoorientational characteristics of these "designer colloids", we have systematically studied the structures, magnetism, and magnetooptical properties of the particles and of their dispersions. We find that these nanostructures require only a fraction of the magnetic field strength of conventional metallomesogens for alignment, and that it is possible to magnetically orient them. This and the fact that the magnetoorientational properties of these novel structures do only weakly depend on particle concentration makes these particles interesting for applications as magnetic actuators, ${ }^{17}$ as steering components in magnetically guided self-propelled devices, ${ }^{5}$ as components in spatial light modulators, ${ }^{24,25}$ or as microscale valves in microfluidic systems. ${ }^{2}$ The detailed structures of these designer colloids and their magnetic and magnetoorientational properties will be presented here, together with an improved synthesis.

\section{Experimental Section}

3-Aminopropyltrimethoxysilane (APS, Gelest) and trinitrobenzenesulfonyl acid (TNBS) (5\% w/v aqueous solution, Aldrich) were used as received. Exfoliated $\left[\mathrm{Bu}_{4} \mathrm{~N}\right]_{x}\left[\mathrm{H}_{1-x} \mathrm{Ca}_{2} \mathrm{Nb}_{3} \mathrm{O}_{10}\right]$ nanosheets were prepared according to Schaak et al., ${ }^{26}$ and 6 $\mathrm{nm}$ oleic acid coated magnetite nanoparticles were synthesized as described by Sun et al. ${ }^{27}$ Anhydrous DMSO was purchased from Fisher Scientific and degassed with nitrogen before use. THF was dried over sodium benzophenone and distilled under nitrogen. Ethanol (95\%) and pyridine (99\%) were used as received. Centrifugations were performed in a Fisher Marathon 21000 centrifuge $(13500 \mathrm{rpm}=21000 \mathrm{~g})$.

APS Ligated $\mathrm{Ca}_{2} \mathrm{Nb}_{3} \mathrm{O}_{10}$ Nanosheets. A suspension of $\left[\mathrm{Bu}_{4} \mathrm{~N}\right]_{x}\left[\mathrm{H}_{1-x} \mathrm{Ca}_{2} \mathrm{Nb}_{3} \mathrm{O}_{10}\right](x=0.2)^{26}$ in DMSO was prepared by dispersing $20 \mathrm{mg}$ of the white solid in $1.0 \mathrm{~mL}$ of anhydrous DMSO after washing it several times with THF to remove residual water. The suspension was stirred under nitrogen (to exclude water) and $30 \mathrm{mg}$ of neat 3-aminopropyltrimethoxysilane (5 M excess) was added. After $1-2 \mathrm{~h}$, stirring was discontinued to allow the larger particles to settle down. The top $75 \%$ of the solution in the flask was collected and centrifuged at $3500 \mathrm{rpm}$ to produce a white solid. The solid was washed three times with a total of $50 \mathrm{~mL}$ of THF, and kept as a wet solid for further use.

Synthesis of $\mathrm{Fe}_{3} \mathrm{O}_{4}-\mathrm{APS}-\mathrm{Ca}_{2} \mathrm{Nb}_{3} \mathrm{O}_{10}$ Clusters. Oleic acidligated magnetite nanoparticles $(5.3 \mathrm{~nm}, 10 \mathrm{mg})$ were dispersed in $1 \mathrm{~mL}$ of THF, and a solution of $20 \mathrm{mg}$ of oleic acid-ligated $\mathrm{Fe}_{3} \mathrm{O}_{4}$ particles was added in $2 \mathrm{~mL}$ of THF. Over $20 \mathrm{~min}, 15$ $\mathrm{mL}$ of ethanol was then added to the rapidly stirred mixture to induce formation of the product. The brown solid was centrifuged off and washed three times with a total of $50 \mathrm{~mL}$ of THF to remove excess $\mathrm{Fe}_{3} \mathrm{O}_{4}$ nanoparticles. The resulting brown solid was kept together with a few drops of residual THF in a closed container. EDS Elemental analysis for $1.0 \mathrm{Ca}_{2} \mathrm{Nb}_{3} \mathrm{O}_{10}$ :1.0 APS: $1.3 \mathrm{Fe}_{3} \mathrm{O}_{4}: 1.5 \mathrm{OA}: 5.0 \mathrm{H}_{2} \mathrm{O}\left(\mathrm{C}_{21} \mathrm{H}_{51} \mathrm{Ca}_{2} \mathrm{Fe}_{3.5} \mathrm{NNb}_{3} \mathrm{O}_{22.5} \mathrm{Si}\right)$. Expected (found): C, 20.02 (19.29); Ca, 6.36 (5.79); Fe, 15.51 (15.60); Nb, 22.12 (22.80); O, 28.57 (27.54); Si, 2.23 (4.34). The discrepancy for $\mathrm{Si}$ is due to the background from the silicon wafer sample holder.

Quantification of Aminopropylsilyl Groups. A 0.01\% (w/ v) aqueous solution of 2,4,6-trinitrobenzene sulfonic acid was prepared by mixing $200 \mu \mathrm{L}$ of $5 \%$ w/v TNBS with $100 \mathrm{~mL}$ of $\mathrm{H}_{2} \mathrm{O}$. Reference solutions with concentrations of $1.54 \times 10^{-3}$, $1.15 \times 10^{-3}, 9.99 \times 10^{-4}, 7.69 \times 10^{-4}$, and $5.39 \times 10^{-4} \mathrm{M}$ of 3-aminopropyltrimethoxysilane were prepared by further diluting a solution of $184 \mathrm{mg}$ in $10 \mathrm{~mL}$ of water. To induce the color reaction, $0.7 \mathrm{~mL}$ of the dye solution was added to $0.3 \mathrm{~mL}$ of the respective APS solutions and the colorless mixture was heated to $80^{\circ} \mathrm{C}$ for $45 \mathrm{~min}$ in a water bath. A UV/vis spectrum was recorded to quantify the absorption of the sample at 422 $\mathrm{nm}$.

To quantify the amine on the sheets, a dispersion of $4.5 \mathrm{mg}$ of amine-modified sheets in $3.0 \mathrm{~mL}$ of solution was prepared and ultrasonicated for $20 \mathrm{~min}$. Then $0.7 \mathrm{~mL}$ of a solution of the dye was added to $0.3 \mathrm{~mL}$ of the nanosheet dispersion and the reaction mixture was heated to $80{ }^{\circ} \mathrm{C}$. After $45 \mathrm{~min}$ a yellow color appeared and a white solid was deposited at the bottom of the container. The solid was centrifuged off, and the absorbance of the supernatant was recorded at $422 \mathrm{~nm}$. Comparison with the reference samples yielded a molar ratio of amine to $\mathrm{Ca}_{2} \mathrm{Nb}_{3} \mathrm{O}_{10}$ of $0.43 \pm 0.06$ indicating an approximate composition of $\mathrm{TBA}_{0.6}\left[\mathrm{NH}_{2} \mathrm{C}_{3} \mathrm{H}_{6} \mathrm{Si}_{0.4} \mathrm{Ca}_{2} \mathrm{Nb}_{3} \mathrm{O}_{10}\right.$ of the material.

Magnetic Measurements. Magnetic measurements were carried out with a Quantum Design Superconducting Quantum Interference Device (SQUID) magnetometer. To determine the anisotropic magnetic properties, the nanosheets were placed on a planar Si wafer $\left(5.0 \times 5.5 \mathrm{~mm}^{2}\right)$, which according to electron microscopy contained $>90 \%$ of the sheets coplanar to the wafer surface. The wafer was mounted inside the SQUID either parallel or perpendicular to the magnetic field.

Optical Measurements. Birefringence patterns were recorded with a home-built glass cuvette with a path length of $1.0 \mathrm{~mm}$. Two pieces of polarizing film (NT38-495 from Edmund Optics) with greater than $99 \%$ polarizing efficiency and $0.029 \mathrm{~mm}$ thickness were mounted to both sides of the cuvette. The curvette was filled with the sample solution and placed in front of a $20 \mathrm{~W}$ neon light source. Light scattering patterns were recorded by placing the sample solution into a standard quartz cuvette with a $1 \mathrm{~cm}$ path length. Illumination was provided by a portable laser with $532 \mathrm{~nm}$ and $5 \mathrm{~mW}$ output. The scattering image was projected onto a screen, $5 \mathrm{~cm}$ away from the sample. Patterns were photographed with a 3.0 Mpixel digital camera. The image resolution and contrast were reduced to enhance the pattern. UV/vis spectra were recorded in standard quartz cuvettes with a Hewlett-Packard 8450A UV-vis spectrometer. For polarized spectra two pieces of the polarizing film were mounted before and after the sample container.

Electron Microscopy. Samples for electron microscopy (scanning electron microscope, SEM, FEI XL30-SFEG) were prepared by drop-coating the dispersion of the respective nanoparticle composites onto $25-\mathrm{mm}^{2}$ pieces of a silicon wafer or on aluminum stubs. Elemental analyses were performed with an EDAX energy-dispersive X-ray spectroscopy system that was 

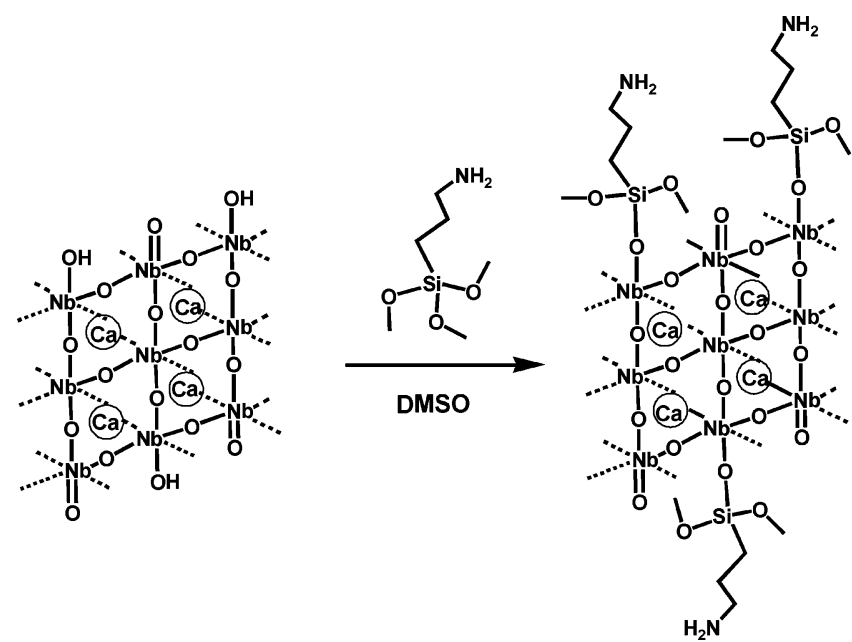

Figure 2. Covalent modification of $\mathrm{HCa}_{2} \mathrm{Nb}_{3} \mathrm{O}_{10}$ nanosheets. The nanosheet is shown as a projection perpendicular to the sheet plane.

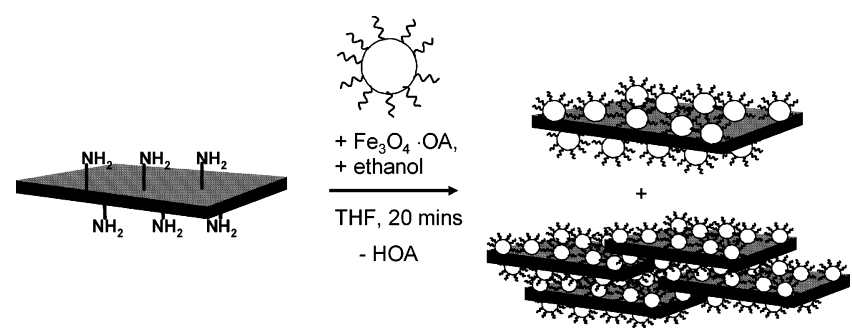

Figure 3. Synthesis of $\mathrm{Fe}_{3} \mathrm{O}_{4}-\mathrm{Ca}_{2} \mathrm{Nb}_{3} \mathrm{O}_{10}$ single clusters and their stacked aggregates.

connected to the SEM. For transmission electron microscopy (Philips CM12), samples were prepared by drop-coating the sample solutions onto holey carbon copper grids (TedPella). HRTEM images were obtained on a Philips CM200 FEG microscope operated at $200 \mathrm{kV}$.

\section{Results and Discussion}

The reaction of $\mathrm{HCa}_{2} \mathrm{Nb}_{3} \mathrm{O}_{10}$ nanosheets ${ }^{26}$ with an excess of 3-aminopropyltrimethoxysilane (APS) in anhydrous DMSO at room temperature produces aminoalkylsilane-modified $\mathrm{Ca}_{2}$ $\mathrm{Nb}_{3} \mathrm{O}_{10}$ nanosheets (Figure 2). This coupling is analogous to the reaction of trialkoxysilanes with silica surfaces in that it requires hydroxyl groups on the $\mathrm{Ca}_{2} \mathrm{Nb}_{3} \mathrm{O}_{10}$ nanosheets.

The amount of aminoalkylsilane in the final product was determined by reaction of the nanosheets with trinitrobenzene- sulfonic acid (TNBS) in water. In the presence of primary amines, TNBS undergoes nucleophilic substitution of the sulfonic acid functionality to form a secondary amine, which can be detected spectroscopically by following the absorbance at $422 \mathrm{~nm} .{ }^{28}$ To quantify the amines, the nanosheets were reacted with the dye for $45 \mathrm{~min}$ in $80^{\circ} \mathrm{C}$ hot water, after which a white solid and a yellow supernatant containing the dye had formed. This indicates that the silane detaches from the sheet during the reaction with the dye. The absorption of the supernatant at $422 \mathrm{~nm}$ was then compared to a linear calibration curve (Supporting Information) that had been obtained by reacting the TNBS dye with variable concentrations of free APS in water. We thus obtained an amine content of $0.43( \pm 0.06) \%$ in the sample, suggesting that amine functionalities are present on $21.5 \%$ of all surface $\mathrm{Nb}-\mathrm{O}$ sites in the sheets. This value corresponds to about half of the protonated $\mathrm{Nb}-\mathrm{O}$ sites $(40-$ $42.5 \%)$ in the exfoliated nanosheets, which follows from the elemental composition of the material $\mathrm{TBA}_{x}\left[\mathrm{H}_{1-x} \mathrm{Ca}_{2} \mathrm{Nb}_{3} \mathrm{O}_{10}\right]_{x}$ $(x=0.15-0.20)$ as reported by Schaak et al. ${ }^{26}$ This supports the assumption that only the protonated sites can react with the silane agent. While it is difficult to assess the detailed binding geometry of the silanes, geometric considerations show that it is impossible for one silicon atom to simultaneously bind to more than one $\mathrm{Nb}-\mathrm{O}$ site on the $\mathrm{Ca}_{2} \mathrm{Nb}_{3} \mathrm{O}_{10}$ nanosheet. We therefore assume that the silanes are bonded only to one $\mathrm{Nb}-\mathrm{O}$ site, and that the two other coordination sites of $\mathrm{Si}$ remain occupied by hydroxy or methoxy groups. The monodentate binding of the silane is supported by the lability of the silane, as evidenced by the easy detachment of the silane during reaction with the TNBS dye. The stability of $\mathrm{SiO}_{2}$-bonded organosilanes had earlier been found to correlate with the number of $\mathrm{Si}-\mathrm{O}$-bonds to the substrate. ${ }^{29}$ IR spectra of the dried nanosheets (Supporting Information) show bands at 1050-1150 $\mathrm{cm}^{-1}$ for $\mathrm{Si}-\mathrm{O}-\mathrm{C}$ and $\mathrm{Si}-\mathrm{O}-\mathrm{Nb}$ that confirm attachment of silane to the nanosheets. However, from these data it is not possible to deduce the number of $\mathrm{Si}-\mathrm{O}-\mathrm{Nb}$ bonds or the nature of the other Si-substituents, alkoxy or hydroxyl. The presence of the aminosilanes also leads to an increase of the solubility of the nanosheets in nonpolar solvents: In contrast to the original particles, the ligated sheets can be dispersed in THF without loss of their liquid crystalline properties that are characteristic for exfoliated sheets from the layered niobates. ${ }^{30}$

Due to the presence of the primary amine groups the nanosheets now possess the ability to bind other inorganic nanomaterials. The title compound forms when ethanol is added to a stirred mixture of excess of oleic acid ligated $\mathrm{Fe}_{3} \mathrm{O}_{4}$
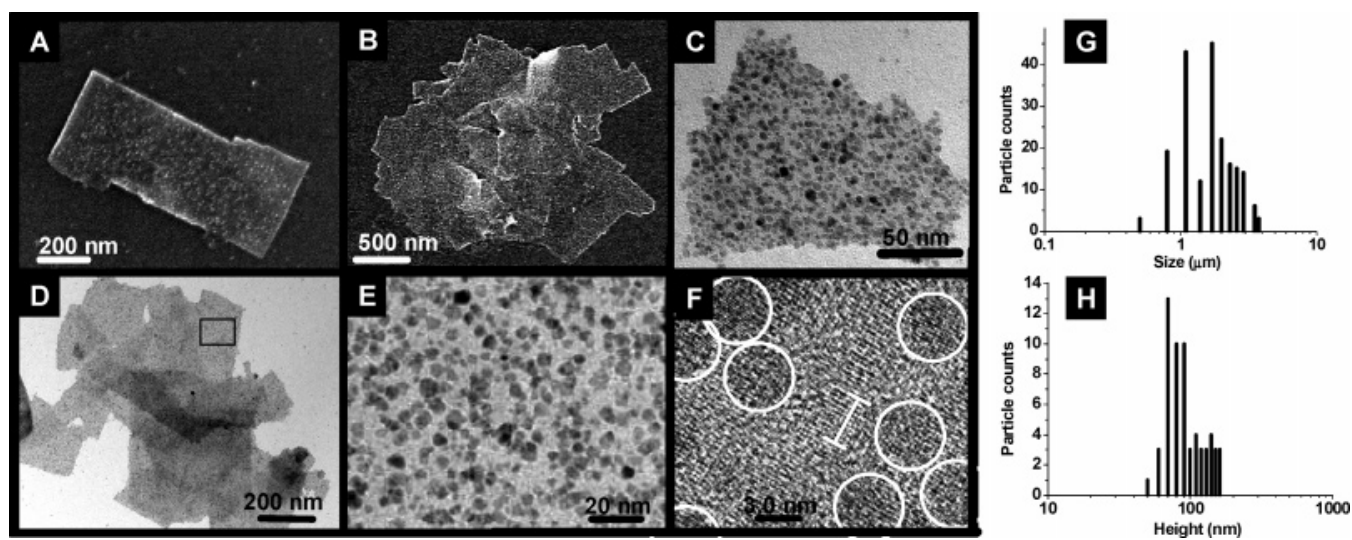

Figure 4. Scanning and transmission electron micrographs of $\mathrm{Fe}_{3} \mathrm{O}_{4}-\mathrm{Ca}_{2} \mathrm{Nb}_{3} \mathrm{O}_{10}$ single clusters and their stacked aggregates. (A) SEM of a single cluster, (B) SEM of a stacked aggregate, (C) TEM of a single cluster, (D) TEM of a cluster aggregate, (E) TEM of a magnified region in D, (F) HRTEM image of a single cluster showing the crystallinity of the sheet and a few magnetite particles as slightly darker circled spots, and (G/H) size and thickness distribution of $\mathrm{Fe}_{3} \mathrm{O}_{4}-\mathrm{Ca}_{2} \mathrm{Nb}_{3} \mathrm{O}_{10}$ stacked aggregates. 


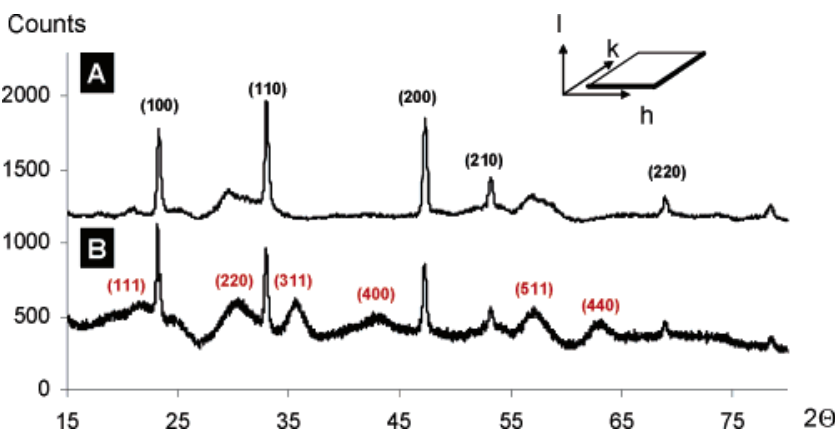

Figure 5. X-ray diffraction pattern recorded for APS $-\mathrm{Ca}_{2} \mathrm{Nb}_{3} \mathrm{O}_{10}(\mathrm{~A})$ and for the $\mathrm{Fe}_{3} \mathrm{O}_{4}-\mathrm{Ca}_{2} \mathrm{Nb}_{3} \mathrm{O}_{10}$ clusters (B) as dried powders. Peak assignments in $\mathrm{A}$ and $\mathrm{B}$ are for $\mathrm{Ca}_{2} \mathrm{Nb}_{3} \mathrm{O}_{10}$ and $\mathrm{Fe}_{3} \mathrm{O}_{4}$, respectively.

nanoparticles $(5.3 \pm 1.0 \mathrm{~nm})$ and of the ligated nanosheets in $\mathrm{THF}$ at room temperature over the course of $20 \mathrm{~min}$ (see Figure 3 ). The product is isolated by centrifugation and purified by repeatedly washing it with tetrahydrofurane to remove excess magnetite. We found that ethanol is essential for the linkage reaction and believe that it helps in removing the oleic acid from the magnetite surface by protonation $\left[\mathrm{p} K_{\mathrm{a}}(\right.$ ethanol $)=$ $15.84]^{31}$ or by solvation.

Scanning and transmission electron micrographs of the product particles (Figure 4) reveal that the formed magnetite$\mathrm{Ca}_{2} \mathrm{Nb}_{3} \mathrm{O}_{10}$ clusters are composed of nanosheets that are coated on both sides with $\mathrm{Fe}_{3} \mathrm{O}_{4}$ particles with densities of $(9.3 \pm 0.5)$ $\times 10^{3}$ particles per $1.0 \mu \mathrm{m}^{2}$ on each side of the sheet. Many of the $\mathrm{Fe}_{3} \mathrm{O}_{4}$ particles appear in groups of closely spaced particles with separations less than $1.0 \mathrm{~nm}$, suggesting that van der Waals interactions and dipolar magnetic interactions influence the assembly. The average surface-to-surface distance of $\mathrm{Fe}_{3} \mathrm{O}_{4}$ nanocrystals on the sheets is $1.6 \pm 0.5 \mathrm{~nm}$.

Depending on the solvent, the single magnetite $-\mathrm{Ca}_{2} \mathrm{Nb}_{3} \mathrm{O}_{10}$ clusters have the ability to form stacked aggregates. Weakly coordinating solvents such as ethanol and THF lead to stacking over the course of minutes, while the strongly coordinating solvent pyridine prevents the $\mathrm{Fe}_{3} \mathrm{O}_{4}: \mathrm{Ca}_{2} \mathrm{Nb}_{3} \mathrm{O}_{10}$ clusters from stacking. While individual clusters can be as small as $0.4 \times$ $1.02 \mu \mathrm{m}^{2}$ and as thin as $21.3 \mathrm{~nm}$ (thicknesses from AFM, Supporting Information), the stacked aggregates have average lateral sizes of $1.6 \pm 0.7 \mu \mathrm{m}$ and thicknesses of $79 \pm 30 \mathrm{~nm}$ (Figure 4G/H). The observed log-normal distributions of the lateral sizes and the thicknesses are typical for pseudo-twodimensional colloidal particles. ${ }^{30}$

Chemically the magnetite-nanosheet composites are quite robust. For example, magnetite particles cannot be removed by repeated treatment of the cluster particles with THF, in which oleic acid-coated magnetite nanoparticles are otherwise soluble.
However, slow detachment of $\mathrm{Fe}_{3} \mathrm{O}_{4}$ nanoparticles occurs in pyridine, and the reaction of the nanoparticle clusters with oleylamine (9-octadecenylamine) at $80{ }^{\circ} \mathrm{C}$ leads to complete detachment of $\mathrm{Fe}_{3} \mathrm{O}_{4}$ nanoparticles within $30 \mathrm{~min}$. These findings indicate that $\mathrm{Fe}_{3} \mathrm{O}_{4}$ are individually connected to $\mathrm{Ca}_{2}$ $\mathrm{Nb}_{3} \mathrm{O}_{10}$ through covalent interactions between probably ferrous/ ferric ions on the nanoparticle surfaces and terminal 3-aminopropylsilyl groups on the sheets.

The bonded $\mathrm{Fe}_{3} \mathrm{O}_{4}$ particles contain residual oleic acid ligands on their surfaces which is apparent from both the intense $\mathrm{C}-\mathrm{H}$ stretching bands in the IR spectra on the nanocomposite (Supporting Information) and the high carbon content of the clusters. Energy dispersive spectra (EDS) suggest $\mathrm{C}_{21} \mathrm{H}_{51} \mathrm{Ca}_{2-}$ $\mathrm{Fe}_{3.5} \mathrm{NNb}_{3} \mathrm{O}_{22.5} \mathrm{Si}$ as the composition of the clusters, which corresponds to a ratio of $1.0 \mathrm{Ca}_{2} \mathrm{Nb}_{3} \mathrm{O}_{10}: 1.0$ APS:1.3 $\mathrm{Fe}_{3} \mathrm{O}_{4}: 1.5$ $\mathrm{OA}: 5.0 \mathrm{H}_{2} \mathrm{O}$ of the components.

HRTEM and X-powder spectra show that the structural integrity of both the $\mathrm{Fe}_{3} \mathrm{O}_{4}$ and APS-modified $\mathrm{Ca}_{2} \mathrm{Nb}_{3} \mathrm{O}_{10}$ nanosheets is maintained in the composite. Figure 4F shows a HRTEM image of a single sheet coated with magnetite particles (visible as dark circular areas). The (100) lattice planes for the $\mathrm{Ca}_{2} \mathrm{Nb}_{3} \mathrm{O}_{10}$ nanosheet of tetragonal symmetry are visible $(0.368$ $\mathrm{nm}$ ) and in good agreement with the crystallographic data for the parent compound $0.387 \pm 0.001 \mathrm{~nm} .^{32}$

$\mathrm{X}$-ray powder diffraction patterns of the covalently modified $\mathrm{Ca}_{2} \mathrm{Nb}_{3} \mathrm{O}_{10}$ sheets and of the magnetite- nanosheet clusters are shown in Figure 5. The sharp reflections in part A belong to intra-sheet reflections of the exfoliated sheets, ${ }^{33}$ while reflections from planes with indices $(00 l)$ are either broad or not observed because the periodicity in the $c$ direction is lost after exfoliation of the sheets from the solid. In addition to these peaks, the pattern for the $\mathrm{Fe}_{3} \mathrm{O}_{4}-\mathrm{Ca}_{2} \mathrm{Nb}_{3} \mathrm{O}_{10}$ adduct (B) contains broad peaks that can be assigned to magnetite.

The magnetic properties were determined for films of $\mathrm{Fe}_{3} \mathrm{O}_{4}-$ $\mathrm{Ca}_{2} \mathrm{Nb}_{3} \mathrm{O}_{10}$ nanosheets on a silicon wafer. In these films the majority $(>90 \%)$ of the particles are aligned with the surface of the wafer. The temperature dependence of the magnetic moments in a 20 Oe field applied parallel to the nanosheets is shown in Figure 6A, after zero field cooling (ZFC) and field cooling (FC). A blocking temperature of about $150 \mathrm{~K}$ is seen, above which the nanoparticles become superparamagnetic. At low temperatures, the nanoparticles and in turn the whole particle-decorated nanosheets are ferrimagnetic and produce a hysteresis (Figure 6B). By changing the orientation of the wafer with respect to the external field, it is possible to distinguish the magnetic behavior for a direction parallel and perpendicular to the plane of the plates. While for both orientations the sample is ferrimagnetic at $5 \mathrm{~K}$ with a coercivity of over 300 Oe (Figure
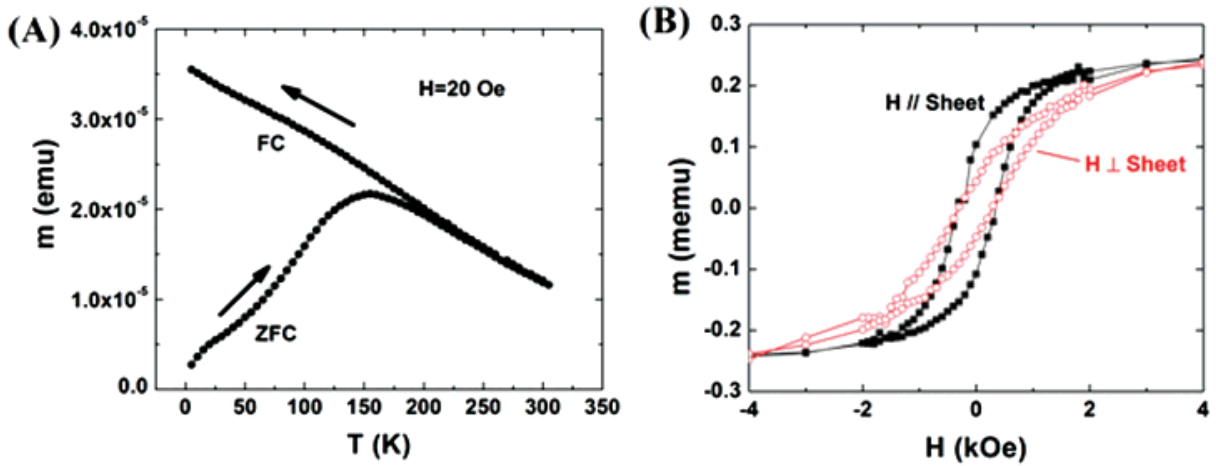

Figure 6. (A) Temperature dependence of the magnetic moment of $\mathrm{Fe}_{3} \mathrm{O}_{4}-\mathrm{Ca}_{2} \mathrm{Nb}_{3} \mathrm{O}_{10}$ nanosheets measured in a 20 Oe field applied parallel to the nanosheets, after zero field cooling (ZFC) and field cooling (FC). (B) Magnetic hysteresis loop of $\mathrm{Fe}_{3} \mathrm{O}_{4}-\mathrm{Ca}_{2} \mathrm{Nb}_{3} \mathrm{O}_{10}$ nanosheets at $5 \mathrm{~K}$, with the applied field parallel (solid squares) or perpendicular (open circle) to the nanosheets. 

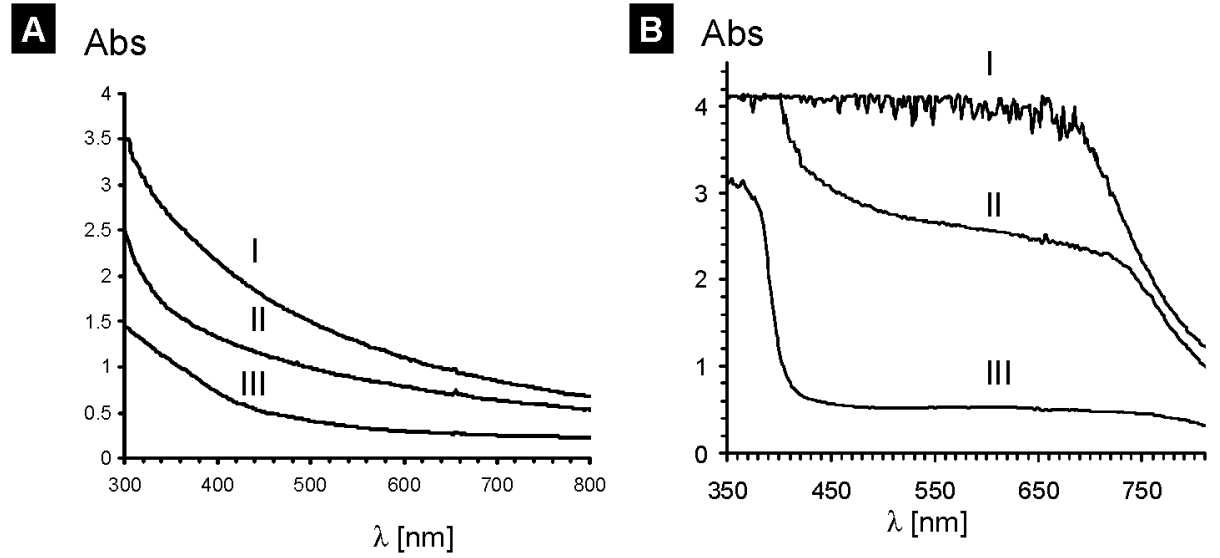

Figure 7. (A) UV/vis spectra of (I) $\mathrm{Fe}_{3} \mathrm{O}_{4}-\mathrm{Ca}_{2} \mathrm{Nb}_{3} \mathrm{O}_{10}$ clusters, (II) APS ligated nanosheets, and (III) Oleic acid-ligated $\mathrm{Fe}_{3} \mathrm{O}_{4}$ particles (all in THF). (B) UV/vis spectra of a dispersion of $\mathrm{Fe}_{3} \mathrm{O}_{4}-\mathrm{Ca}_{2} \mathrm{Nb}_{3} \mathrm{O}_{10}$ between two cross-oriented polarizers: (I) $\mathrm{H}=0$, (II) $\mathrm{H}$ parallel to polarizer, and (III) polarizing film only.

$6 \mathrm{~B})$, the observed loop is slanted in perpendicular field, and the nanosheets are found to saturate at a higher field than that in the parallel geometry. Also, in the perpendicular geometry, the remanence is only $18 \%$ of the saturation magnetization, much lower than the $42 \%$ remanence in the parallel geometry, a factor of 2.3 difference. This is direct evidence of a magnetic anisotropy of the nanosheets, and it indicates that the easy axis occurs in the plane of the nanosheets. The observed anisotropy is analogous to the shape anisotropy that is typically associated with magnetic thin films. ${ }^{34}$ For a magnetic film, a perpendicular field needs to compensate for the demagnetization field before saturating the film, whereas an in-plane field does not. Thus the saturation field in the perpendicular geometry is higher than that in the parallel geometry by $4 \pi M_{\mathrm{S}}$, where $M_{\mathrm{S}}$ is the saturation magnetization. The magnetic plates investigated here behave similarly, because the spacing between adjacent $\mathrm{Fe}_{3} \mathrm{O}_{4}$ nanoparticles is small, allowing for dipolar interactions between adjacent particles.

The effects of the dipole fields due to the neighboring magnetic particles on a particular site can be estimated. Each of the magnetite nanoparticles can be approximated as a magnetic dipole with size $d=5.3 \mathrm{~nm}$, and the arrangements of these particles on the nanosheets can be approximated by a square array of dipoles with a center-to-center spacing of $l \sim 7$ $\mathrm{nm}$. When the magnetic moments of all the particles are aligned by an in-plane field applied parallel to the nanosheets, the moment of a particular particle is stabilized by the vector sum of the dipole fields from all its neighbors, which is about 0.4 kOe. When the magnetic moments of all the particles are aligned by an out-of-plane field applied perpendicular to the nanosheets, the dipole field from one particle on another always tends to destabilize the moment. The vector sum is about $-0.8 \mathrm{kOe}$, i.e., the external field needs to overcome a $0.8 \mathrm{kOe}$ net dipolar field before aligning the moments. In the present samples with spherical nanoparticles of magnetite, the above saturation fields in the parallel and perpendicular field geometries will be further modified, e.g., to take into account demagnetization fields due to the shape of the particles. Nonetheless, this difference in saturation field of the order of $1 \mathrm{kOe}$ is in good agreement with the observed anisotropy seen in Figure 6B.

The UV/vis spectra of free $\mathrm{Fe}_{3} \mathrm{O}_{4}$ particles, of ligated nanosheets, and of the cluster particles are characterized by broad featureless absorptions over nearly the entire range of the visible spectrum (Figure 7). Solutions of oleic acid-supported $\mathrm{Fe}_{3} \mathrm{O}_{4}$ nanoparticles are black because of electronic transitions between the $\mathrm{Fe} 3 \mathrm{~d}$ bands in the material. ${ }^{35}$ In contrast,
A

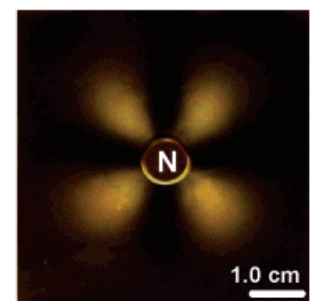

B

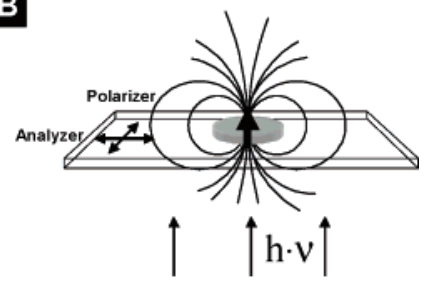

Figure 8. (A) Photograph of a suspension of $\mathrm{Fe}_{3} \mathrm{O}_{4}-\mathrm{Ca}_{2} \mathrm{Nb}_{3} \mathrm{O}_{10}(1.6$ $\pm 0.7 \mu \mathrm{m}$ mean edge length) in pyridine between two crossed polarizers under the influence of an inhomogeneous magnetic field (6000 Oe at the center). (B) Schematic drawing showing the orientation of polarizer and analyzer, and the direction of the magnetic field lines with respect to the sample.
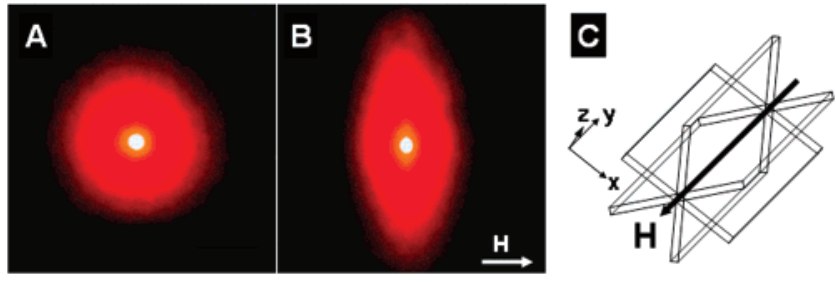

Figure 9. Light scattering from a nanosheet suspension $(1.6 \pm 0.7$ $\mu \mathrm{m}$ mean sizes) in THF exposed to zero (A) or 200 Oe (B) magnetic fields (an arrows indicates the magnetic field direction). The sample dispersion was contained in a quartz cuvette with $1 \mathrm{~cm}$ path length and the incident light (532 $\mathrm{nm}$ laser) was linearly polarized with vertical polarization direction. (C) Schematic view of the orientation of the $\mathrm{Fe}_{3} \mathrm{O}_{4}-\mathrm{Ca}_{2} \mathrm{Nb}_{3} \mathrm{O}_{10}$ clusters with the field lines.

dispersions of the nanosheets appear white due to scattering by the micrometer sized particles in THF. Dispersions of the $\mathrm{Fe}_{3} \mathrm{O}_{4}-\mathrm{Ca}_{2} \mathrm{Nb}_{3} \mathrm{O}_{10}$ clusters reflect the optical properties of the building blocks and thus appear brownish and turbid.

Because the colloidal plates have a magnetic anisotropy, it is possible to align the plates in solution with the aid of a relatively weak magnetic field ( 200 Oe). Dispersions of such magnetically aligned particles acquire an optical anisotropy, which manifests in asymmetric light scattering and birefringence patterns. These patterns can be used to obtain information about the orientations of the particles in the dispersion. The birefringence pattern obtained for a dispersion of $\mathrm{Fe}_{3} \mathrm{O}_{4}-\mathrm{Ca}_{2} \mathrm{Nb}_{3} \mathrm{O}_{10}$ particles in pyridine between two cross-oriented polarizers and under the influence of a magnetic field is shown in Figure 8. In the experimental configuration the magnetic field lines radially penetrate the sample as shown in Figure 8B. The light intensity transmitted through the crossed polarizers and the sample varies 


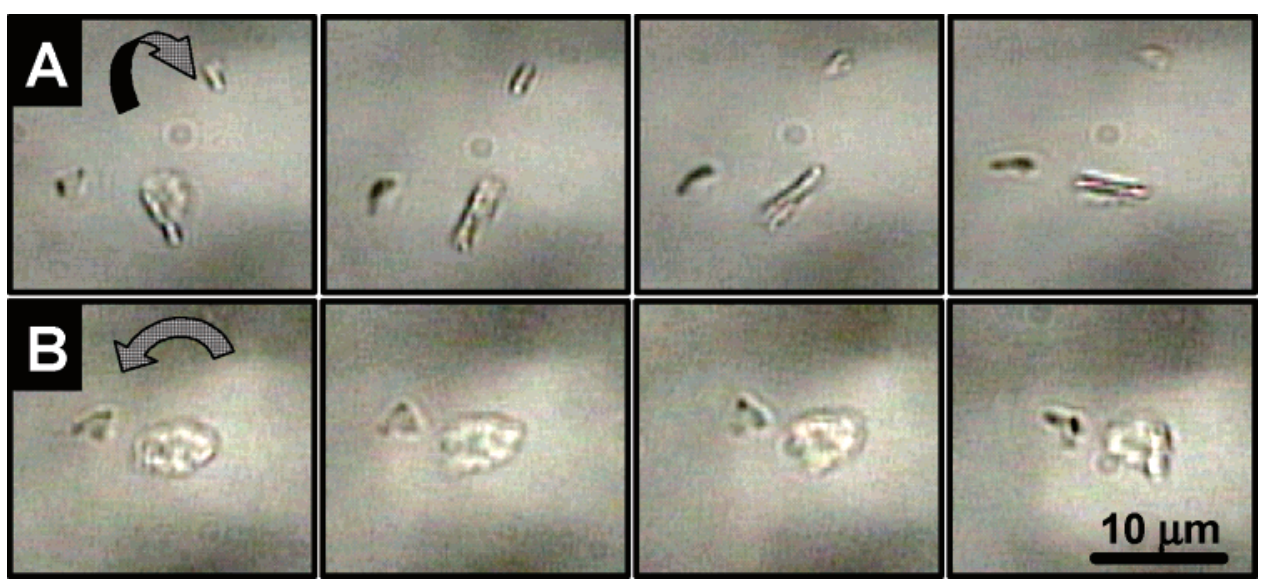

Figure 10. Magnetic control of the orientation of a cluster aggregate in THF: (A) Rotation around an axis parallel to the plate and (B) rotation around an axis perpendicular to the plate. The scale bar is $10 \mu \mathrm{m}$.

according to a $I \sim \sin ^{2}(2 \theta)$ dependence with $\theta$ being the azimuthal angle in the plane perpendicular to the direction of light propagation. This pattern is characteristic of dispersions of aligned colloidal particles 36,37 and it indicates that the colloidal plates align with their principal optical and magnetic axes either parallel or perpendicular with respect to the magnetic field lines.

Figure 7B also shows the absorption spectra taken for both the dark and light regions in the pattern of Figure 8A. The depolarization effect is observable over nearly the entire visible spectrum, but remains strongest in the $450-700 \mathrm{~nm}$ region. At wavelengths $\lambda<400 \mathrm{~nm}$ the effect is limited by the absorption of the polarizing film (see spectrum III). At $\lambda>750 \mathrm{~nm}$ the polarizing ability of the polarizing film is greatly reduced, leading to the observed decrease of the absorption.

To obtain the absolute orientation of the plates we have also conducted light scattering and optical microscopy measurements on dispersions of the colloidal plates. Figure 9 shows light scattering patterns of dispersion of the magnetic plates $(1.6 \pm$ $0.7 \mu \mathrm{m}$ large particles) in THF when exposed to monochromatic laser radiation $(\lambda=532 \mathrm{~nm})$. In the absence of a magnetic field the scattering pattern is symmetric as expected for a dispersion of randomly orientated particles. When a 200 Oe magnetic field is applied with the field lines perpendicular to the propagation direction of the incident light, the scattering pattern becomes asymmetric with maximum scattering angles of $51^{\circ}$ and $25.5^{\circ}$, perpendicular and parallel to the magnetic field lines, respectively. The asymmetric scattering pattern indicates a preferential orientation of the magnetic plates with regard to the magnetic field lines. It is difficult to determine the absolute orientation of the plates from the scattering pattern because Mie scattering theory, which applies in this case, provides no analytical solutions for platelike colloidal particles. However, comparison with scattering patterns obtained for dispersions of aligned colloidal plates, ${ }^{38}$ colloidal rods, ${ }^{20,39}$ and liquid crystals ${ }^{21,40-42}$ suggests that in the present case the colloidal plates align with one of their two principal geometrical (and optical) axes with the magnetic field lines, as shown in Figure 9C.

Optical microscopy on colloidal dispersions provides direct evidence for the magnetic orientation of the particles. Figure 10 shows two colloidal plates $\left(6.8 \times 5.4 \mu \mathrm{m}^{2}\right.$ and $2.8 \times 3.2$ $\mu \mathrm{m}^{2}$ ) freely dispersed in THF, under the influence of a $500 \mathrm{Oe}$ inhomogeneous magnetic field (a movie in mpeg format is available in the Supporting Information).

In the first row of the figure, the plates are rotated around an in-plane axis ( $y$ as defined in Figure 1B) by rotating the external magnetic field around the $y$ axis. In the second row of the figure, the particles are rotated around an out-of-plane axis $(z)$ by rotating the magnetic field around the $z$ axis. These combined observations demonstrate that the magnetic plates align their magnetic easy axes (either $x$ or $y$ ) with the magnetic field. Magnetic fields can also be used to rotate these biaxial structures in solution via the torque exerted on the magnetite nanoparticles. In terms of their magnetic orientation, the plates behave analogously to magnetic rods with uniaxial magnetic anisotropy.

\section{Conclusion}

We demonstrated here a simple method for the large-scale synthesis of biaxial magnetic microstructures composed of $\mathrm{Ca}_{2}-$ $\mathrm{Nb}_{3} \mathrm{O}_{10}$ nanosheets and $\mathrm{Fe}_{3} \mathrm{O}_{4}$ nanoparticles. Magnetic measurements show that the inorganic structures are superparamagnetic at room temperature, and that they have a biaxial magnetic anisotropy that arises from magnetic dipole interactions between the magnetite nanoparticles along the two principal directions of these pseudo-two-dimensional structures. The magnetic anisotropy makes it possible to align and rotate these microstructures with a weak magnetic field. In terms of their magnetooptical properties the compounds synthesized here are similar to liquid crystals except that they are much more sensitive to magnetic fields. The magnetic fields required for orientation are by $1-2$ orders of magnitude lower than those used to align metallomesogens $(4-20 \mathrm{kOe})^{9}$ and by $2-3$ orders of magnitude lower than those used for diamagnetic liquid crystals $(2-20 \mathrm{~T}) .{ }^{43}$ The optical and magnetic properties make these superparamagnetic colloidal plates interesting for optical applications, e.g. magnetic liquid crystal displays. The fact that the magnetoorientational properties of the synthesized superparamagnetic plates are inherent to the particles and independent of the particle concentration enables applications of such structures as valves in microfluidic devices, as reflective components in spatial light modulators and waveguides, ${ }^{24,25}$ as steering components in magnetically guided self-propelled devices and probes (e.g. in medicine), ${ }^{5,44}$ or as magnetic labels to investigate the flow of liquids.

Acknowledgment. This work has been supported by the University of California-Davis (startup and CLE funds) and the Petroleum Research Fund (38057-G5). We thank Dr. William Casey in the Department of Land Air and Water Resources for use of a Digital Imaging Multimode AFM, which was purchased with NSF-EAR94-14103. We also thank Dr. Susan Kauzlarich for use of high-temperature furnaces, and the staff and facilities at the National Center for Electron Microscopy for support. 
Supporting Information Available: UV/vis spectra and calibration curves for the quantification of aminopropylsilyl groups, IR spectra, AFM data, and a movie sequence (mpeg2) that shows the magnetic field-controlled orientation of the superparamagnetic plates. This material is available free of charge via the Internet at http://pubs.acs.org.

\section{References and Notes}

(1) Feynman, R. P. Eng. Sci. 1960, 33.

(2) Craighead, H. G. Science 2000, 290, 1532.

(3) Bingelyte, V.; Leach, J.; Courtial, J.; Padgett, M. J. Appl. Phys. Lett. 2003, 82, 829 .

(4) Park, C.; Robertson, R. E. Mater. Sci. Eng. A 1998, 257, 295.

(5) Paxton, W. F.; Kistler, K. C.; Olmeda, C. C.; Sen, A.; St. Angelo, S. K.; Cao, Y.; Mallouk, T. E.; Lammert, P. E.; Crespi, V. H. J. Am. Chem. Soc. 2004, 126, 13426.

(6) Gimenez, R.; Lydon, D. R.; Serrano, J. L. Curr. Opin. Solid State Mater. Sci. 2002, 6, 527.

(7) Spoliansky, D.; Ponsinet, V.; Ferre, J.; Jamet, J. P. Eur. Phys. J. A 2000, 1, 227.

(8) Binnemans, K.; Gorller-Walrand, C. Chem. Rev. 2002, 102, 2303. (9) Binnemans, K.; Galyametdinov, Y. G.; Van Deun, R.; Bruce, D. W.; Collinson, S. R.; Polishchuk, A. P.; Bikchantaev, I.; Haase, W.; Prosvirin, A. V.; Tinchurina, L.; Litvinov, I.; Gubajdullin, A.; Rakhmatullin, A.; Uytterhoeven, K.; Van Meervelt, L. J. Am. Chem. Soc. 2000, 122, 4335.

(10) Love, J. C.; Urbach, A. R.; Prentiss, M. G.; Whitesides, G. M. J. Am. Chem. Soc. 2003, 125, 12696.

(11) Urbach, A. R.; Love, J. C.; Prentiss, M. G.; Whitesides, G. M. J. Am. Chem. Soc. 2003, 125, 12704.

(12) Lee, H.; Purdon, A. M.; Chu, V.; Westervelt, R. M. Nano Lett. 2004, 4, 995.

(13) Blakemore, R. Science 1975, 190, 377.

(14) Philipse, A. P.; Maas, D. Langmuir 2002, 18, 9977.

(15) Bentley, A. K.; Trethewey, J. S.; Ellis, A. B.; Crone, W. C. Nano Lett. 2004, 4, 487.

(16) Martin, J. I.; Nogues, J.; Liu, K.; Vicent, J. L.; Schuller, I. K. J. Magn. Magn. Mater. 2003, 256, 449.

(17) Zahn, M. J. Nanopart. Res. 2001, 3, 73.

(18) Dierking, I. Textures of Liquid Crystals; Wiley-VCH: Weinheim, Germany, 2003.

(19) Hultgren, A.; Tanase, M.; Chen, C. S.; Reich, D. H. IEEE Trans. Magn. 2004, 40, 2988.
(20) Lemaire, B. J.; Davidson, P.; Ferre, J.; Jamet, J. P.; Panine, P.; Dozov, I.; Jolivet, J. P. Phys. Rev. Lett. 2002, 8812, 5507.

(21) Bellomo, E. G.; Davidson, P.; Imperor-Clerc, M.; Deming, T. J. J. Am. Chem. Soc. 2004, 126, 9101.

(22) Osterloh, F. E. J. Am. Chem. Soc. 2002, 124, 6248.

(23) Liu, K.; Zhao, L.; Klavins, P.; Osterloh, F. E.; Hiramatsu, H. J. Appl. Phys. 2003, 93, 7951.

(24) Tabiryan, N. V.; Nersisyan, S. R. Appl. Phys. Lett. 2004, 84, 5145.

(25) Sayyah, K.; Wu, C.-S.; Wu, S.-T.; Efron, U. Appl. Phys. Lett. 1992, 61,883 .

(26) Schaak, R. E.; Mallouk, T. E. Chem. Mater. 2000, 12, 2513.

(27) Sun, S. H.; Zeng, H. J. Am. Chem. Soc. 2002, 124, 8204.

(28) Habeeb, A. F. S. A. Anal. Biochem. 1966, 14, 328.

(29) Waddell, T. G.; Leyde, D. E.; DeBello, M. T. J. Am. Chem. Soc. 1981, 103, 5303.

(30) Miyamoto, N.; Nakato, T. J. Phys. Chem. B 2004, 108, 6152.

(31) DeTar, D. F. J. Am. Chem. Soc. 1982, 104, 7205.

(32) Jacobsen, A. J.; Johnson, J. W.; Lewandowski, J. T. Inorg. Chem. 1985, 24, 3727.

(33) Ebina, Y.; Sasaki, I.; Watanabe, A. Solid State Ionics 2002, 151, 177.

(34) Cullity, B. D. Introduction to Magnetic Materials; Addison-Wesley Publishing Co.: Reading, MA, 1972.

(35) Park, S. K.; Ishikawa, T.; Tokura, Y. Phys. Rev. B 1998, 58, 3717.

(36) Larson, R. G. The Structure and Rheology of Complex Fluids; Oxford University Press: New York, 1999.

(37) Dierking, I. Textures of Liquid Crystals; Wiley-VCH: Weinheim, Germany, 2003.

(38) Gabriel, J. C. P.; Camerel, F.; Lemaire, B. J.; Desvaux, H.; Davidson, P.; Batail, P. Nature 2001, 413, 504.

(39) Gabriel, J. C. P.; Davidson, P. Adv. Mater. 2000, 12, 9.

(40) Safinya, C. R.; Sirota, E. B.; Bruinsma, R. F.; Jeppesen, C.; Plano, R. J.; Wenzel, L. J. Science 1993, 261, 588.

(41) Firestone, M. A.; Tiede, D. M.; Seifert, S. J. Phys. Chem. B 2000, 104, 2433.

(42) Yao, H. S.; Kagoshima, Y.; Kitamura, S.; Isohashi, T.; Ozawa, Y.; Kimura, K. Langmuir 2003, 19, 8882.

(43) Christianen, P. C. M.; Shklyarevskiy, I. O.; Boamfa, M. I.; Maan, J. C. Phys. B (Amsterdam, Neth.) 2004, 346, 255.

(44) Polla, D. L.; Erdman, A. G.; Robbins, W. P.; Markus, D. T.; DiazDiaz, J.; Rizq, R.; Nam, Y.; Brickner, H. T.; Wang, A.; Krulevitch, P. Аnnи. Rev. Biomed. Eng. 2000, 2, 551. 